Nuclear Physics B264 (1986) 423-436

(c) North-Holland Publishing Company

\title{
LARGE WIDTH IN THE LEE MODEL FOR THE HIGGS-GOLDSTONE SECTOR
}

\author{
David N. WILLIAMS \\ The Harrison M. Randall Laboratory of Physics, The University of Michigan, \\ Ann Arbor, Michigan 48109, USA
}

Received 10 June 1985

We discuss a variant of the unstable particle Lee model motivated by the problem of large width in Higgs meson decay. The essential result is that, in the point-interaction model, the width for decay into Goldstone bosons (W's and Z's), defined from the second-sheet unstable particle pole, obeys

$$
\Gamma_{0} \leqslant \Gamma<\frac{3}{2} \Gamma_{0}
$$

where $\Gamma_{0}$ is the naive perturbative width, and the ordering is from weak coupling at the lower end to strong coupling at the upper end. If the point interaction of the Lee model is modified to make the tree scattering amplitude for Goldstone particles relativistically invariant, a large-coupling upper bound for the width results,

$$
\Gamma<10.7 m_{\mathrm{H}},
$$

where by comparison, the naive perturbative width grows like $m_{\mathrm{H}}^{3}$.

We find that defining the renormalized coupling at the complex Higgs pole is reasonable in the Lee model, even when the pole moves far from the real axis. Furthermore, scattering effects remain strong, in the sense that the unitarity bound is roughly approached over a large range of energies, although the scattering amplitude never reaches more than about $80 \%$ of saturation in the point-interaction model, contrary to naive perturbation theory. We also point out, without discussing it in this paper, what appears to be a new fact about the Lee model, that the Higgs propagator has a bound-state pole in addition to the resonance pole.

\section{Introduction}

The Lee model [1] for unstable particles transports the Weisskopf-Wigner [2] two-level model to a Fock space setting, and in some sense it is the concrete example of a number of unstable particle ideas, including those of second-sheet poles, discrete energy dissolved into the continuum, unitary time evolution with deviations from exponential decay, exact $S$-matrix unitarity, and mass, coupling constant, and wave function renormalization.

It has a number of drawbacks. It is not relativistically invariant, because it throws away significant pieces of the local interaction; it does not have crossing, and in our 
case has only s-wave scattering. It has the curious history that for a long time it was thought to suffer from ghosts, until Ynduráin [3] noticed that ghosts are an artifact of no-recoil kinematics.

The present study is motivated by the large-width problem for Higgs bosons with large mass, which makes it questionable how they are to be observed [4]. We cannot claim any definitive insights, because the Lee model we consider for the HiggsGoldstone sector is a drastic truncation of the underlying gauge field theory. But the model can at least give hints about certain speculations, for example, concerning the interaction of large coupling with violation of the perturbative unitarity bound. We also believe that, because of its pedagogical interest as an unstable particle field theory, the Lee model deserves to be spelled out more than it has been, either in the literature in general or in this paper in particular.

We specify the model precisely in sect. 2 ; for now we state that the fundamental processes allowed in the Lee model interaction are

$$
H \leftrightarrow W_{+} W_{-}, Z Z
$$

where $H$ is a scalar (Higgs) meson with mass $m_{\mathrm{H}}$, and $\left(W_{+}, Z, W_{-}\right)$is an isotopic triplet of scalar (Goldstone) mesons with small or zero mass $m_{\mathrm{W}}$ (in particular, $\left.m_{\mathrm{H}}>2 m_{\mathrm{W}}\right)$. In this paper, we take $m_{\mathrm{W}}$ strictly zero, because it simplifies the analysis, and the difference between zero and small mass should be irrelevant for large coupling, when large coupling is tied to large Higgs mass and the concern is with large energies. The price to be paid is that there is a bound-state situation that gets obscured, as we shall explain shortly.

With $m_{\mathrm{w}}$ strictly zero, we describe our results as follows:

(i). In the point interaction version, let $\Gamma_{0}$ be the leading-order perturbation expression for the Higgs width, and $\frac{1}{2} \Gamma$ the exact, negative imaginary part of the Higgs propagator pole in the energy plane. We prove that there is exactly one pole in the lower right quadrant of the resonance sheet. Then for all values of the bare coupling,

$$
\Gamma_{0} \leqslant \Gamma<\frac{3}{2} \Gamma_{0}
$$

Here $\Gamma$ has a monotone-increasing dependence on the bare coupling (which is finite in the Lee model), and the upper limit becomes saturated in the strong coupling limit.

Perturbation theory in the Higgs sector of the Weinberg-Salam model suggests that as $m_{\mathrm{H}}$ becomes large there is a perturbative violation of unitarity, and that the Higgs width might grow like $m_{\mathrm{H}}^{3}$ [5]. One could then wonder whether enforcement of unitarity might prevent the width from growing as fast as suggested by perturbation theory [4], but the above result shows that unitarity does not of itself have that effect. In the exactly unitary, point-interaction Lee model, the width grows just like perturbation theory suggests. 
(ii). In a non-point-interaction version, which includes a form factor chosen to make the tree scattering amplitude for Goldstone particles agree with that of the fully relativistic sigma model from which the Lee model is truncated, the scaled Higgs width has an upper bound at large coupling,

$$
\Gamma / 2 m_{\mathrm{H}}<5.35 \text {. }
$$

In that case, the width does not grow as fast as the perturbative expression. There is some support for this phenomenon in an interesting large-coupling treatment by M.B. Einhorn [6], based on the large- $N$ expansion, which gets a bound of 1 instead of 5.35 .

(iii). In both versions of the Lee model, there is a bound-state pole in the Higgs propagator below the $W_{+} W_{-}$or $Z Z$ threshold, in case those particles are given a mass, or at negative energy, if the masses are zero, in addition to the second-sheet resonance pole. At small coupling, both the existence and the non-analytic behavior of the bound-state pole position can be obtained correctly at one-loop order. These facts have led us to wonder whether a bound-state pole might not also appear in the Higgs propagator in the relativistic sigma model (with non-zero Goldstone masses) from which our Lee model is truncated, at one-loop order. We have confirmed that that is indeed the case, for small coupling and small $m_{\mathrm{W}} / m_{\mathrm{H}}$.

Whether the one-loop signal really means a bound state in the sigma model is unclear to us at this point. In the Lee model with relativistic kinematics, the presence of the bound state appears to be a new fact. The fact that it occurs at negative energy when $m_{\mathrm{W}}$ is zero is an embarrassment to the stability of the vacuum, but we do not take it seriously for our application, because of our motivation from large energy. The existence of a bound state does support that the force in the $I=0$ channel is attractive, as Lee, Quigg, and Thacker [5] pointed out (at large $m_{\mathrm{H}}$ ).

This paper focuses on the unstable particle pole, and omits any details about bound states.

(iv). Since the Lee model is exactly soluble with non-trivial renormalization, we can ask what insight it affords into non-perturbative renormalization. In particular, the unstable Higgs particle has a non-trivial, although finite, wave function renormalization, which reflects the fact that, in the stable case, the dressing transformation from the bare, unperturbed one-particle state is non-trivial.

There has been some equivocation in the literature about wave function renormalization for unstable particles. We adopt the attitude that the wave function renormalization should normalize the residue at the second-sheet pole in the Higgs two-point function. One could wonder whether that remains meaningful when the width grows large and the pole moves far from the real axis.

If the renormalized coupling is defined in terms of the value of the renormalized three-point function (one Higgs and two Goldstone fields) at some point in momentum space, the Higgs wave function renormalization explicitly feeds into it. In that 
approach, one has to keep the normalization point away from any bound-state energy, to avoid sensitivity in the renormalized coupling.

A second approach is to define the renormalized coupling in terms of the residue of the Higgs pole in the four-point function for Goldstone fields. This avoids any direct mention of the Higgs wave function renormalization, and the normalization point is "natural".

The result in either case, for the point interaction, is that the renormalized coupling grows linearly at large bare coupling (in particular, large bare coupling is large renormalized coupling). Since nothing strange happens, it seems reasonable to normalize at the pole, even though the pole moves far from the physical region.

In the form factor case, the renormalized coupling saturates to a finite value at large bare coupling. Again, this strikes us as reasonable, because the width does that, too.

Indeed, in both cases it would seem reasonable to trade the renormalized coupling for the width as a fundamental parameter.

We display the four-point function approach in sect. 5, but we omit the correspondence with the three-point function.

By contrast, in the stable Higgs case, there is no extra bound-state pole; and neither the sensitivity question nor that of the plausibility of normalizing far from the physical region arises. The well-known result [3], which we do not discuss further in this paper, is that the renormalized coupling saturates to a finite value at large bare coupling, for a point interaction.

(v). Although the unstable particle pole moves far from the physical region at large coupling, its effects in the amplitude for scattering of its decay products do not disappear. The physical resonance signal, where the phase shift rises through $\frac{1}{2} \pi$ as the energy increases on the real axis, whereupon the partial wave unitarity bound gets saturated, still occurs in a rough approximation, even though the pole is far from the real axis. What happens is that the phase shift rises more slowly and lingers, near about $51^{\circ}$ for the point interaction, over an ever larger interval of energy. The unitarity bound is never really saturated; the scattering amplitude comes to a little less than $80 \%$ of saturation, contrary to naive perturbation theory. In that sense, strong coupling has strong effects in the scattering. The effect is not so dramatic, but is still present, in the form factor version.

This phenomenon was pointed out earlier in Einhorn's large- $N$ treatment [6]. It is of course a simple idea, that strong coupling means large scattering effects. It tempts one to ask, if large width is indeed due to large coupling, whether there might not be a way of disentangling such a broad Higgs signal from those of the competing processes that are left out by the Lee model.

In general, we do not treat the form factor interaction in much detail. We do not expect either version to have much phenomenological validity at large coupling. We have included some discussion of both interactions because they provide examples of quite different behaviors at large coupling. We have focused on the point interaction 
because it is analytically simpler. Also in the interest of simplicity, and because they are essentially the same for the point interaction, we have used the bare rather than the renormalized coupling as the fundamental parameter.

\section{Definition of the model}

At large Higgs mass, the Higgs-Goldstone sector of the Weinberg-Salam model is expected to dominate Higgs decay [5,7]; that is, only physical Higgs particles, $H$ 's, and longitudinal $W_{\mathrm{L}}$ 's and $Z_{\mathrm{L}}$ 's count. Then we invoke the "scalar dominance theorem" $[5,7]$ for large $m_{\mathrm{H}}$ that amplitudes involving $\left(H, W_{\mathrm{L}_{ \pm}}, Z_{\mathrm{L}}\right)$ are the same as the corresponding amplitudes involving $\left(H, W_{ \pm}, Z\right)$, where $\left(W_{ \pm}, Z\right)$ are Goldstone scalars, up to terms of order $m_{\mathrm{W}} / \sqrt{s}$, for $s \gg m_{\mathrm{W}}^{2}, m_{\mathrm{Z}}^{2}$.

The model at this point has a massless, Goldstone boson isotopic triplet $\Phi_{\alpha}$, with $\Phi_{ \pm 1}=W_{ \pm}, \Phi_{0}=Z$, and a massive, neutral scalar $H$, with interaction density

$$
V_{\mathrm{I}}=\lambda v H\left(\Phi_{\alpha} \Phi_{\alpha}^{*}+H^{2}\right)+\frac{1}{4} \lambda\left(\Phi_{\alpha} \Phi_{\alpha}^{*}+H^{2}\right)^{2}
$$

where the vacuum expectation value $v$, the Fermi constant $G_{\mathrm{F}}$, the coupling $\lambda$, and the Higgs mass $m_{\mathrm{H}}$ are related by

$$
\frac{1}{v^{2}}=2 G_{\mathrm{F}}, \quad \lambda=\frac{1}{2} G_{\mathrm{F}} m_{\mathrm{H}}^{2} .
$$

Although they will shortly become irrelevant, we mention here our conventions for isotopic spin. The lower index on $\Phi_{\alpha}$ labels the charge destroyed by $\Phi_{\alpha}$. We take $\Phi_{\alpha}$ to obey a convention like the complex conjugate of a spherical harmonic. Spherical harmonics normally have their indices up. The raising operator for odd integer spin differs by a sign from complex conjunction, so

$$
\Phi^{\alpha}=(-1)^{1-\alpha} \Phi_{-\alpha}=-\Phi_{\alpha}^{*}
$$

and $-\Phi^{\alpha}$ has the same convention as a spherical harmonic. The expression in terms of hermitian fields is therefore

$$
\Phi_{+}=-\sqrt{\frac{1}{2}}\left(\Phi_{1}-i \Phi_{2}\right), \quad \Phi_{-}=\sqrt{\frac{1}{2}}\left(\Phi_{1}+i \Phi_{2}\right), \quad \Phi_{0}=\Phi_{3}
$$

The Lee model truncation is to drop the quartic terms and the $H^{3}$ term in (2.1), and furthermore to restrict the fundamental interactions to $H \leftrightarrow\left(W_{+} W_{-}, Z Z\right)$. The resulting model can be described in terms of Fock space creation and destruction operators $a, a^{*}$ for the Higgs and $b_{\alpha}, b_{\alpha}^{*}$ for the Goldstone triplet. We take 
relativistic normalizations:

$$
\begin{array}{rlrl}
{\left[a(\boldsymbol{k}), a\left(\boldsymbol{k}^{\prime}\right)^{*}\right]} & =2 \omega_{H} \delta\left(\boldsymbol{k}-\boldsymbol{k}^{\prime}\right), & & \omega_{\mathrm{H}}^{2}=m_{\mathrm{H}}^{2}+\boldsymbol{k}^{2}, \\
{\left[b_{\alpha}(\boldsymbol{k}), b_{\beta}\left(\boldsymbol{k}^{\prime}\right)^{*}\right]} & =2 \omega \delta\left(\boldsymbol{k}-\boldsymbol{k}^{\prime}\right) \delta_{\alpha \beta}, & \omega^{2}=m_{\mathrm{W}}^{2}+\boldsymbol{k}^{2} .
\end{array}
$$

We eventually put the renormalized mass $m_{\mathrm{W}}$ to zero, and we put $m_{\mathrm{H}}$ at the observed Higgs level position, the real part of the second-sheet pole in the energy plane at $\boldsymbol{k}=0$.

The hamiltonian is

$$
\begin{aligned}
H & =H_{\mathrm{H}}+\delta H_{\mathrm{H}}+H_{\mathrm{W}}+V \\
H_{\mathrm{H}}+\delta H_{\mathrm{H}} & =\int \frac{\mathrm{d}^{3} k}{2 \omega_{\mathrm{H}}} a^{*} a\left(\omega_{\mathrm{H}}+\delta \omega_{\mathrm{H}}\right) \\
H_{\mathrm{W}} & =\int \frac{\mathrm{d}^{3} k}{2 \omega} b_{\alpha}^{*} b_{\alpha} \omega \\
V & =V_{+}+V_{-}, \\
V_{+} & =\frac{-\lambda v}{(2 \pi)^{3 / 2}} \int \frac{\mathrm{d}^{3} k}{2 \omega_{H}} \frac{\mathrm{d}^{3} k_{1}}{2 \omega_{1}} \frac{\mathrm{d}^{3} k_{2}}{2 \omega_{2}} b_{\alpha}\left(\boldsymbol{k}_{1}\right) b^{\alpha}\left(\boldsymbol{k}_{2}\right) a^{*}(\boldsymbol{k}) \delta\left(\boldsymbol{k}-\boldsymbol{k}_{1}-\boldsymbol{k}_{2}\right) \eta \\
V_{+} & =V_{-}^{*} .
\end{aligned}
$$

The factor $\eta$ will be put to one in the usual, point-interaction situation. The modification that we consider is

$$
\eta=\sqrt{\frac{2 \omega_{\mathrm{H}}}{\omega_{1}+\omega_{2}+\omega_{H}}},
$$

which has the effect of making the tree scattering amplitude for Goldstone particles relativistically invariant, and may be thought to put back in part of the effect of the relativistic Higgs propagator, which gets truncated into an "old-fashioned perturbation theory" propagator in the point interaction version.

Here is a review of the renormalization.

The mass parameters $m_{\mathrm{H}}$ and $m_{\mathrm{W}}$ are renormalized quantities. The Higgs counterterm $\delta \omega_{\mathrm{H}}$ is chosen to make the real part of the second-sheet energy pole in the Higgs two-point function equal to the renormalized energy parameter $\omega_{H}$. It is 
logarithmically divergent in the point-interaction case and finite for our modified $\eta$. The creation and destruction operators and the coupling parameters $\lambda_{v}$ are bare quantities.

There is no vacuum self-energy renormalization, because the bare and physical vacua are the same, the Fock vacuum for the bare destruction operators.

There is no Goldstone mass renormalization, because the bare one-particle Goldstone states are eigenstates of $H$.

$$
H b_{\alpha}(\boldsymbol{k})^{*}|\mathrm{vac}\rangle=\omega b_{\alpha}(\boldsymbol{k})^{*}|\mathrm{vac}\rangle .
$$

Since the bare and dressed Goldstone one-particle states coincide, there is also no Goldstone wave function renormalization.

We have already described our attitude towards the Higgs wave function renormalization, which is finite.

\section{Reduction to the decay sector}

The hamiltonian leaves the sector of Fock space spanned by $a^{*}|v a c\rangle$ and $b_{\alpha}^{*} b_{\beta}^{*}|v a c\rangle$ invariant. Furthermore, the isotopic structure of the interaction makes it vanish on the isotopic triplet subspace of that sector. There is non-trivial interaction only in the "decay sector", spanned by $a^{*}|\mathrm{vac}\rangle$ and $b_{\alpha}^{*} b^{\alpha *}|\mathrm{vac}\rangle=$ $\Sigma_{\alpha}(-1)^{1-\alpha} b_{\alpha}^{*} b_{-\alpha}^{*}|\mathrm{vac}\rangle$.

We choose a basis of momentum eigenstates in the decay sector as follows:

$$
\begin{aligned}
|\boldsymbol{k}\rangle & =a^{*}(\boldsymbol{k})|\mathrm{vac}\rangle, \quad\left\langle\boldsymbol{k} \mid \boldsymbol{k}^{\prime}\right\rangle=2 \omega_{\mathrm{H}} \delta\left(\boldsymbol{k}-\boldsymbol{k}^{\prime}\right), \\
\left|\boldsymbol{k}_{1}, \boldsymbol{k}_{2}\right\rangle & =-\frac{1}{\sqrt{2 \cdot 3}} b_{\alpha}^{*}\left(\boldsymbol{k}_{1}\right) b^{\alpha *}\left(\boldsymbol{k}_{2}\right)|\mathrm{vac}\rangle \\
\left\langle\boldsymbol{k}_{1}^{\prime}, \boldsymbol{k}_{2}^{\prime} \mid \boldsymbol{k}_{1}, \boldsymbol{k}_{2}\right\rangle & =\frac{1}{2} 2 \omega_{1} 2 \omega_{2}\left[\delta\left(\boldsymbol{k}_{1}^{\prime}-\boldsymbol{k}_{1}\right) \delta\left(\boldsymbol{k}_{2}^{\prime}-\boldsymbol{k}_{2}\right)+\delta\left(\boldsymbol{k}_{1}^{\prime}-\boldsymbol{k}_{2}\right) \delta\left(\boldsymbol{k}_{2}^{\prime}-\boldsymbol{k}_{1}\right)\right] .
\end{aligned}
$$

The interaction is then determined by

$$
\left\langle\boldsymbol{k}_{1}, \boldsymbol{k}_{2}|V| \boldsymbol{k}\right\rangle=g \eta \delta\left(\boldsymbol{k}-\boldsymbol{k}_{1}-\boldsymbol{k}_{2}\right), \quad g \equiv \sqrt{2 \cdot 3} \frac{\lambda v}{(2 \pi)^{3 / 2}} .
$$

At this point, the problem has been reduced to an equivalent problem with two scalar particles and no isospin; the only vestige of isotopic spin is the factor $\sqrt{3}$ in eq. (3.2).

The theory is solved in the decay sector once the resolvent operator $(z-H)^{-1}$ is known for all complex $z$. In the Lee model, all matrix elements can be expressed in 
terms of

$$
\begin{aligned}
\left\langle\boldsymbol{k}^{\prime}\left|\frac{1}{z-H}\right| \boldsymbol{k}\right\rangle & =2 \omega_{\mathrm{H}} \delta\left(\boldsymbol{k}^{\prime}-\boldsymbol{k}\right) G_{k}(z) \\
D(z) & \equiv G_{k}(z)^{-1} \\
& =z-\omega_{\mathrm{H}}-\delta \omega_{\mathrm{H}}-\frac{1}{2 \omega_{\mathrm{H}}} g^{2} \int \frac{\mathrm{d}^{3} k_{1}}{2 \omega_{1}} \frac{\mathrm{d}^{3} k_{2}}{2 \omega_{2}} \eta^{2} \frac{1}{z-\omega_{1}-\omega_{2}} \delta\left(\boldsymbol{k}-\boldsymbol{k}_{1}-\boldsymbol{k}_{2}\right)
\end{aligned}
$$

Initially, the denominator function $D(z)$ is defined in a cut $z$-plane (the physical, or first sheet) with a cut on the positive real axis from $E_{\mathrm{T}}$ to $\infty$. Although we do not discuss them here, one should remain alert to the possibility of real zeros in $D$ below the threshold $E_{\mathrm{T}}$. The integral in $D$ can be simplified by the standard relativistic transformation to Gårding-Wightman variables [8]:

$$
\begin{aligned}
\frac{\mathrm{d}^{3} k_{1}}{2 \omega_{1}} \frac{\mathrm{d}^{3} k_{2}}{2 \omega_{2}} & =\mathrm{d}^{4} P \mathrm{~d} \Omega \sqrt{\frac{s-4 m_{\mathrm{W}}^{2}}{s}} \frac{\theta\left(s-4 m_{\mathrm{W}}^{2}\right)}{8}, \\
s & \equiv P \cdot P=\left(\omega_{1}+\omega_{2}\right)^{2}-\left(\boldsymbol{k}_{1}+\boldsymbol{k}_{2}\right)^{2}, \\
D(z) & =z-\omega_{\mathrm{H}}-\delta \omega_{\mathrm{H}}+\int_{E_{\mathrm{T}}}^{\infty} \mathrm{d} E \frac{f(E)}{E-z}, \\
f(E) & =\frac{\pi g^{2}}{4 \omega_{\mathrm{H}}} \eta^{2} \sqrt{\frac{E^{2}-E_{\mathrm{T}}^{2}}{E^{2}-\boldsymbol{k}^{2}}}, \quad E_{\mathrm{T}}^{2} \equiv \boldsymbol{k}^{2}+4 m_{\mathrm{W}}^{2}, \\
\eta^{2} & =1, \quad \text { point interaction, } \\
& =\frac{2 \omega_{\mathrm{H}}}{E+\omega_{\mathrm{H}}}, \quad \text { modified interaction. }
\end{aligned}
$$

For simplicity, we put $m_{\mathrm{w}}=0$ from now on. Then the square root threshold factor in $f(E)$ becomes unity, and, in the point interaction case, $f \equiv f(E)$ is constant. We also work at $\boldsymbol{k}=0$, so the lower limit of integration in (3.4c) is $E_{\mathrm{T}}=0$. From now on, we write $m=m_{\mathrm{H}}$ and $\delta m=\delta m_{\mathrm{H}}$. For our purposes, it is convenient to study $D(z)$ in the plane cut along the negative $z$-axis, chosen so that the upper half plane, excluding the real axis, is common with that of the physical sheet. We call this the "resonance sheet", to indicate that it contains the resonance zero. A 
bound-state zero of $D$ below threshold would be found on the cut of the resonance sheet by approaching the real axis from above.

In the point-interaction case, we introduce a cutoff, to be removed shortly, in the upper limit of the integral in eq. (3.4c), to make its real part finite. We fix $\delta m$ so that the resonance zero is at $z_{0}=m-i \gamma$, where $m>0$ is fixed, and $\gamma=\frac{1}{2} \Gamma>0$ depends on the bare coupling. It will be clear in sect. 4 that we then have a single zero in the right half of the resonance sheet (including the positive real axis). With the cutoff, we have

$$
\begin{gathered}
D(z)=z-m-\delta m+f\left[i \pi+\ln \frac{\kappa-z}{z}\right], \\
\delta m=f \ln \left|\frac{\kappa-z_{0}}{z_{0}}\right|, \quad-\pi<\operatorname{Im} \ln <\pi .
\end{gathered}
$$

When the cutoff is removed, $\kappa \rightarrow \infty$, we get on the resonance sheet,

$$
D(z)=z-m+f\left[i \pi-\ln \frac{z}{|m-i \gamma|}\right],
$$

with the same definition of the argument in the imaginary part of the logarithm.

No cutoff procedure is necessary for the modified interaction, which gives

$$
\begin{aligned}
D(z) & =z-m-\delta m+\frac{2 m f}{z+m}\left[i \pi-\ln \frac{z}{m}\right], \\
\delta m & =\operatorname{Re} \frac{2 m f}{z_{0}+m}\left[i \pi-\ln \frac{z_{0}}{m}\right] .
\end{aligned}
$$

\section{The Higgs width}

As we said earlier, our discussion will be more detailed for the point interaction than for the form factor version.

\subsection{POINT INTERACTION}

Let $z=x+i y$ be in the right half of the resonance sheet. The equation for zeros of $D$ in the right half resonance sheet is

$$
z=m+f\left[\ln \left|\frac{z}{m-i \gamma}\right|-i \pi+i \tan ^{-1} \frac{y}{x}\right], \quad-\frac{1}{2} \pi<\tan ^{-1}<\frac{1}{2} \pi
$$


There are no zeros above or on the real axis in the right half plane, because the imaginary part of the right side is non-vanishing and negative.

We have chosen $\delta m$ to give a resonance zero with real part at $m, z_{0}=(m-i \gamma)$, if we can find the negative imaginary part $\gamma$. The equation for $\gamma>0$ is

$$
\frac{\gamma}{m}=\frac{f}{m}\left[\pi+\tan ^{-1} \frac{\gamma}{m}\right]=\frac{f}{m}\left[\frac{3 \pi}{2}-\tan ^{-1} \frac{m}{\gamma}\right]
$$

Clearly

$$
\pi \leqslant \gamma / f \leqslant \frac{3}{2} \pi
$$

The leading order expression for small $f$ is

$$
\gamma_{0}=\pi f=\frac{3}{16 \pi^{2}} \lambda^{2} v^{2}=\frac{3}{64 \pi^{2}} v^{2} G_{\mathrm{F}}^{2} m^{4} .
$$

It is straightforward to see that the graphs of the functions of scaled width $\gamma / \mathrm{m}$ defined by the left and right sides of eq. (4.2), for fixed $f / m$, have one and only one intersection for $\gamma / m>0$. Furthermore,

$$
\frac{\mathrm{d} \gamma}{\mathrm{d} f}=\frac{\pi+\tan ^{-1}(\gamma / m)}{1-f m /\left(m^{2}+\gamma^{2}\right)},
$$

which shows that $\mathrm{d} \gamma / \mathrm{d} f>0$, because $\gamma \geqslant \pi f$ means $m^{2}+\gamma^{2} \geqslant 2 \gamma m \geqslant 2 \pi f m \geqslant f m$; so the denominator and numerator are both positive. We conclude that $\gamma$ is a monotone increasing function of $f$ which vanishes like $\gamma_{0}=\pi f$ at $f=0$ and approaches the limit $\gamma / f=\frac{3}{2} \pi$ at $f=\infty$. That proves the claim stated in the introduction in eq. (1.1).

We have found that there exists one and only one resonance zero with real part $m$ for all positive bare couplings $f$. We now show that there are no other resonance zeros in the right half of the resonance sheet.

It is convenient to use variables scaled by $f$ instead of $m$, with reflected imaginary part: $\zeta=\xi+i \eta=(x-i y) / f$. The equation for the imaginary part of a zero of $D$ in the lower right resonance sheet has the same form as eq. (4.2), even if the real part is not at $\xi=m / f$ :

$$
\eta=\pi+\tan ^{-1} \frac{\eta}{\xi}
$$

In the new variables, we label the zero we have found as $\zeta_{0}=(m+i \gamma) / f$. The equation for the real part of a general zero may be cast in the form

$$
\xi-\xi_{0}=\ln |\zeta|-\ln \left|\zeta_{0}\right|
$$


Any zero, including $\zeta_{0}$, must lie at an intersection of the curves in the first quadrant of the $\xi, \eta$ plane defined by eqs. (4.6) and (4.7). The first curve, $\eta=\eta_{1}(\xi)$, obeys

$$
\begin{gathered}
\pi \leqslant \eta \leqslant \frac{3}{2} \pi, \\
\frac{\mathrm{d} \eta_{1}}{\mathrm{~d} \xi}=\frac{-\eta_{1}}{\xi^{2}-\xi+\eta_{1}^{2}} .
\end{gathered}
$$

The inverse function can be solved explicitly, $\xi_{1}=\eta / \tan \eta$. The second curve is

$$
\begin{aligned}
\eta & =\eta_{2}(\xi)=\left[\left|\xi_{0}\right|^{2} \exp 2\left(\xi-\xi_{0}\right)-\xi^{2}\right]^{1 / 2}, \\
\frac{\mathrm{d} \eta_{2}}{\mathrm{~d} \xi} & =\frac{\xi^{2}-\xi+\eta_{2}^{2}}{\eta_{2}} .
\end{aligned}
$$

The factor $\xi^{2}-\xi+\eta^{2}$ in the derivatives has no real zeroes for $\xi>0$ and $\eta>\frac{1}{2}$, so in particular it has none for $\eta \geqslant \pi$. Thus, the first curve always has a negative slope, and the second always has a positive slope, in the region $\eta \geqslant \pi$ where they could intersect.

There is an intersection at $\zeta_{0}$, by construction. There can then be no other intersection, because the monotonicity of their slopes prevents the curves from getting back together again.

We conclude that $\zeta=\zeta_{0}$ is the only solution of eq. (4.7), and that there is a unique resonance in the lower right quadrant of the resonance sheet, whose imaginary part is small at small coupling (and fixed $m$ ) and moves to $-\infty$ at an essentially linear rate as the coupling parameter goes to $\infty$.

Recall that we have been working in the complex energy plane at zero threemomentum. Including a non-zero momentum would certainly not result in a relativistic complex dispersion law for the resonance pole, because the model breaks Lorentz invariance. A similar Lorentz symmetry breaking effect is present in the modified interaction, to which we now turn, although it builds in relativity at the tree level.

\subsection{FORM FACTOR INTERACTION}

In the non-point interaction case, the equation for $\gamma$, the negative imaginary part of the zero, when the real part is $m$, is

$$
\frac{\gamma}{m}=\frac{4 f}{m} \frac{1}{4+(\gamma / m)^{2}}\left\{\pi+\tan ^{-1} \frac{\gamma}{m}-\frac{\gamma}{4 m} \ln \left[1+(\gamma / m)^{2}\right]\right\},
$$

where $f$ is the same constant as before, given by eq. (4.4). To discuss the solution, we 
use scaled variables $\eta=\gamma / m$ and $h=4 f / m$. The existence, uniqueness, and large coupling limit of the solution $\eta(h)$ of eq. (4.10) can be discussed easily and convincingly by plotting a few curves on a small computer, and with a little more work can be made rigorous. We state the results.

The right side of eq. (4.10) has a zero at

$$
0=\pi+\tan ^{-1} \eta-\frac{1}{4} \eta \ln \left(1+\eta^{2}\right),
$$

unique for $0 \leqslant \eta \leqslant \infty$, which is the value

$$
\begin{aligned}
\eta_{\infty} & =\lim _{h \rightarrow \infty} \eta(h), \\
5.34645 & \leqslant \eta_{\infty} \leqslant 5.3465 .
\end{aligned}
$$

The solution $\eta(h)$ of eq. (4.10) is a monotone, increasing function for $0 \leqslant h \leqslant \infty$, obeying $0 \leqslant \eta(h) \leqslant \eta_{\infty}$. This proves the bound stated in eq. (1.2) in the introduction.

We have not studied the question whether there are extra resonance zeros with real part different from $m$.

\section{Renormalized coupling and scattering}

Because we want to discuss the saturation of unitarity, we point out that partial waves can be constructed by standard relativistic techniques, by using the GårdingWightman variables mentioned sketchily in sect. 3, even though the theory is not Lorentz invariant. The only practical difference is that the scattering phase shifts are not functions of $s=E^{2}-p^{2}$ alone, but of $E$ and $p^{2}$ separately. We work as before at $\boldsymbol{p}=0$, and there is only an s-wave phase shift.

Let the scattering matrix be parameterized as $S=I-2 \pi i T$. Then the $W_{+} W_{-}, Z Z$ (Goldstone) scattering amplitude, which is only $I=0$ in our case, can be expressed in terms of the two-particle states defined in eq. (3.1b) as

$$
\begin{aligned}
\left\langle\boldsymbol{k}_{1}^{\prime}, \boldsymbol{k}_{2}^{\prime}|T| \boldsymbol{k}_{1}, \boldsymbol{k}_{2}\right\rangle & =\delta\left(E^{\prime}-E\right)\left\langle\boldsymbol{k}_{1}^{\prime}, \boldsymbol{k}_{2}^{\prime}\left|V \frac{1}{E+i \varepsilon-H} V\right| \boldsymbol{k}_{1}, \boldsymbol{k}_{2}\right\rangle \\
& =\delta\left(E^{\prime}-E\right) \delta\left(\boldsymbol{k}_{1}^{\prime}+\boldsymbol{k}_{2}^{\prime}-\boldsymbol{k}_{1}-\boldsymbol{k}_{2}\right) \frac{|g \eta|^{2}}{2 \omega_{\mathrm{H}}} G_{k}(E+i \varepsilon),
\end{aligned}
$$

where $E=\omega_{1}+\omega_{2}$, where $g$ and $\eta$ are defined in eqs. (3.2) and (3.4e,f), and where we have used the structure of the Lee model interaction to drop a first order term in $V$, and all occurrences of $\delta H_{\mathrm{H}}$ in the numerator. 
The s-wave phase shift at $\boldsymbol{k}_{1}+\boldsymbol{k}_{2}=0$ can be found from

$$
\frac{\mathrm{e}^{2 i \delta(E)}-1}{2 i}=\frac{\pi^{2}}{4 m_{\mathrm{H}}}|g \eta|^{2} G_{k}(E+i \varepsilon) .
$$

We define the renormalized coupling by comparing the exact $G$ to the tree amplitude:

$$
\left\langle\boldsymbol{k}_{1}^{\prime}, \boldsymbol{k}_{2}^{\prime}|T| \boldsymbol{k}_{1}, \boldsymbol{k}_{2}\right\rangle_{\mathrm{tree}}=\delta^{(4)}\left(\boldsymbol{k}_{1}^{\prime}+\boldsymbol{k}_{2}^{\prime}-\boldsymbol{k}_{1}-\boldsymbol{k}_{2}\right) \frac{|g \eta|^{2}}{2 \omega_{\mathrm{H}}} \frac{1}{E+i \varepsilon-\omega_{\mathrm{H}}},
$$

where we have used the tree version of $G^{-1}, D_{\text {tree }}(z)=z-\omega_{\mathrm{H}}$. The residue of the exact $G$ at the resonance pole $z_{0}=(m-i \gamma)$ at zero momentum is found by expanding $D(z)=\left(z-z_{0}\right) D^{\prime}\left(z_{0}\right)+\cdots$; we then define

$$
g_{\text {ren }}^{2}=\frac{g^{2}}{\left|D^{\prime}\left(z_{0}\right)\right|}
$$

For the point interaction, we find at zero momentum

$$
g_{\text {ren }}^{2}=\frac{g^{2}}{|1-f /(m-i \gamma)|} .
$$

Keeping in mind $f=\pi \mathrm{g}^{2} / 4 m$ and the bound in eq. (4.3) on $\gamma$, it is clear that $g_{\mathrm{ren}}^{2}$ is essentially linear in the bare coupling $g^{2}$, as we claimed in the introduction, going to $\frac{3}{2} \pi g^{2}$ as $g^{2} \rightarrow \infty$.

The point-interaction phase shift can be expressed in the physical region, $E>0$, in terms of $f$ :

$$
\frac{\mathrm{e}^{2 i \delta_{0}}-1}{2 i}=\frac{\pi f}{E-m+f[\ln |(m-i \gamma) / E|+i \pi]} .
$$

It is trivial that unitarity is exact. It is also not hard to check that for large $f$ there is no value of $E$ that makes the real part of the denominator in the above equation zero, which would put the scattering amplitude at its maximum size with $\delta_{0}=\frac{1}{2} \pi$. The magnitude of the denominator can, however, be minimized by minimizing the real part, at $E=f$. In the strong coupling limit, that produces

$$
\mathrm{e}^{i \delta_{0}} \sin \delta_{0}=\frac{\pi}{1+\ln \frac{3}{2} \pi+i \pi},
$$

when $f / m$ becomes large. This yields a phase shift $\delta_{0} \approx 51^{\circ}$ at maximum scattering 
in the strong coupling limit. The expression (5.6) shows that the scattering amplitude will remain appreciable over an interval in $E$ of order $f$, for $f$ large.

The renormalized coupling constant for the form factor interaction is obtained by substituting $z=m-i \gamma$ into

$$
D^{\prime}(z)=1-\frac{2 m f}{(z+m)^{2}}\left(1+\frac{m}{z}-\ln \frac{z}{m}+i \pi\right) .
$$

At large $f$, the renormalized coupling saturates to

$$
g_{\text {ren }, \infty}^{2} \cong 4.4 m^{2}
$$

where we put in the limiting value for $\gamma / m$ from eq. (4.12). If we define the renormalized version of the sigma model coupling, $\lambda v$ in eq. (2.1), by the same proportionality factor as in $g_{\text {ren, } \infty}$, we get

$$
(\lambda v)_{\mathrm{ren}, \infty} \cong 13.5 m
$$

Although we think it utterly without phenomenological meaning, we mention that, if the mass and coupling constant relations in eq. (2.2) are taken as renormalized equations, neglecting any radiative corrections, with the usual expression for the Fermi constant in terms of $m_{\mathrm{W}}$ and the Weinberg angle, then eq. (5.10) gives an upper bound $m_{\mathrm{H}} / m_{\mathrm{W}}<100$ at large coupling.

The large coupling phase shift at maximum scattering can be worked out, but the tenuous validity of the model makes it hardly worth the effort. The scattering amplitude should be broad, but not as broad as in the point interaction case.

It is a pleasure to acknowledge that this paper was stimulated by questions posed by Gordon L. Kane, and to thank both him and Martin B. Einhorn for helpful conversations.

\section{References}

[1] T.D. Lee, Phys. Rev. 95 (1954) 1329;

M. Lev́y, Nuovo Cim. 13 (1959) 115, 14 (1960) 612

[2] G. Höhler, Z. Phys. 152 (1958) 542

[3] F.J. Ynduráin, J. Math. Phys. 7 (1966) 1133

[4] G.L. Kane, Windows for new physics at super colliders, Conf. on the physics of the XXI century, Tucson, Arizona (December, 1983), Univ. of Michigan preprint UM TH 83-25

[5] B.W. Lee, C. Quigg, and H.B. Thacker, Phys. Rev. D16 (1977) 1519

[6] M.B. Einhorn, Nucl. Phys. B246 (1984) 75

[7] J.M. Cornwall, D.N. Levin, and G. Tiktopoulos, Phys. Rev. D10 (1974) 1145

[8] A. Wightman, L'invariance dans la méchanique quantique relativiste, Dispersion relations and elementary particles, eds. C. DeWitte and R. Omnès, Ecole d'été de physique théorique, Les Houches, 1960 (Hermann, Paris, 1960) pp. 198, 199 